九州大学学術情報リポジトリ

Kyushu University Institutional Repository

\title{
Importance of gated CT acquisition for the quantitative improvement of the gated PET/CT in moving phantom
}

Sakaguchi, Yuichi

Division of Radiological Science, Department of Health Sciences, Kyushu University

Mitsumoto, Tatsuya

Division of Radiological Science, Department of Health Sciences, Kyushu University

Zhang, Tiejiao

Division of Radiological Science, Department of Health Sciences, Kyushu University

Mitsumoto, Katsuhiko

Radiological Science Course, Department of Health Sciences, School of Medicine, Kyushu University

他

http://hdl. handle. net/2324/26102

出版情報：Annals of Nuclear Medicine. 24 (7)，pp.507-514，2010-08-01. Springer Japan バージョン :

権利関係: (C) The Japanese Society of Nuclear Medicine 2010 
Respiratory Gated PET/CT

ORIGINAL ARTICLE

$<$ TITLE $>$

Importance of gated $\mathrm{CT}$ acquisition for the quantitative improvement of the gated

$\mathrm{PET} / \mathrm{CT}$ in moving phantom

$<$ A shot title $>$

Respiratory Gated PET/CT

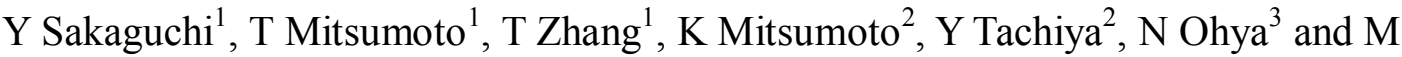
Sasaki ${ }^{1}$

${ }^{1}$ Division of Radiological Science, Department of Health Sciences, Graduate School of Medical Sciences, Kyushu University,

${ }^{2}$ Radiological Science Course, Department of Health Sciences, School of Medicine, Kyushu University,

${ }^{3}$ Department of Medical Technology, Kyushu University Hospital, 
Respiratory Gated PET/CT

Corresponding author and reprint request:

Masayuki Sasaki, MD, $\mathrm{PhD}$,

Division of Radiological Science, Department of Health Sciences, Graduate School of

Medical Sciences, Kyushu University,

3-1-1 Maidashi, Higashi-ku, Fukuoka 812-8582, Japan

Tel: +81-92-642-6746, Fax: +81-92-642-6674

E-mail: msasaki@shs.kyushu-u.ac.jp 


\section{ABSTRACT}

Objective: The aim of this study was to investigate the utility of gated PET/CT and CT attenuation correction (AC) for the quantitation of radioactivity.

Methods: An ellipse phantom containing six spheres, ranging from $10-37 \mathrm{~mm}$ in diameter, was filled with $36.7 \mathrm{kBq} / \mathrm{mL}$ of F-18. The respiratory motion was simulated by a motor-driven plastic platform to move the phantom with a displacement of $2 \mathrm{~cm}$ in the craniocaudal direction at a frequency of 15 per minute. With the phantom at rest, $\mathrm{PET} / \mathrm{CT}$ data were acquired and used as a standard (nonmotion). With the phantom in motion, PET data were acquired in both the static and gated modes (sPET and gPET, respectively). Helical CT (HCT), Slow CT (SCT), Average CT (ACT), and 4-Dimensional CT (4DCT) were acquired and used to correct attenuation. On both PET and CT images, the maximum radioactivity, dimensions, and CT numbers were measured on the central slices.

Results: In nonmotion, recovery coefficients (RC) whose spheres were $22 \mathrm{~mm}$ or smaller gradually decreased. Regarding motion, the PET counts of the spheres in the static acquisition were lower than those acquired in nonmotion with either type of CTAC (sPET-HCT: -43.8\%, sPET-SCT: -51.4\%, sPET-ACT: -49.5). Gated acquisition of PET significantly improved the PET counts (gPET-HCT: $-30.1 \%)(\mathrm{p}<0.05)$, while 
Respiratory Gated PET/CT

additional gated acquisition of CT significantly improved them further (gPET-4DCT:

$-15.2 \%)(\mathrm{p}<0.01)$. The dimensions of sPET were overestimated, but those of gPET were close to the standard values. The SCT significantly overestimated the dimensions, and the water density area decreased $(\mathrm{p}<0.01)$. The 4DCT images were similar to the HCT images.

Conclusions: In respiratory motion, PET acquisition in the static mode underestimated the radioactivity and overestimated the dimensions. Neither SCT nor ACT improved these errors. Although PET acquisition in the gated mode improved the quantification of PET/CT images, the additional gated CT acquisition using 4DCT is required for further improvement.

KEY WORDS: respiration motion; gated; PET/CT; 4DCT; attenuation correction 


\section{INTRODUCTION}

$\mathrm{PET} / \mathrm{CT}$, which provides anatomical and functional information, can be useful for tumor diagnosis, particularly regarding differential diagnosis, staging, response evaluation, and prognosis (1). However, PET emission data acquisition is required for a few minutes per bed and also consists of several respiratory cycles. Therefore, the usual PET images are taken over an averaged state of respiration. The respiration-averaged PET images result in an underestimation of radioactivity and an overestimation of tumor volume in the thoracic and abdominal regions, especially in the vicinity of the diaphragm (2). The underestimation of radioactivity increases the incidence of false-negative results. Some faint accumulations are not visible on images, and the standardized uptake values (SUV) of some lesions become low. Furthermore, the difference in temporal resolution results in a spatial misalignment between PET and CT data $(3,4)$.

To eliminate spatial misalignment between PET and CT images in motion objects, the averaging of CT images over respiratory phases has been proposed. Slow $\mathrm{CT}$ (SCT) is acquired with a long gantry rotation time that includes several respiratory cycles $(5,6)$. Average CT (ACT) images were developed by averaging the CT images in 
Respiratory Gated PET/CT

all respiratory phases observed by 4-Dimensional CT (4DCT) acquisition in the cine mode (7). These blurred CT images are reported to minimize misregistration between the CT attenuation correction (CTAC) and PET data acquired in static mode $(6,7)$. Another method is gated acquisition to eliminate the effect of respiratory motion for PET emission data (8). In the gated-acquisition protocol, PET emission data are continuously acquired in list mode as respiratory motion is monitored. The data are divided into several respiratory phases and are used to reconstruct PET/CT images of each respiratory phase. In addition, 4DCT was developed to better register CT and PET data acquired in gated mode (9). 4DCT is acquired in cine mode using the respiratory system and is divided into phases similar to those of gated PET (10). However, the effect of the combination of PET acquisition mode and CT attenuation correction has not yet been evaluated.

The purpose of this study is to assess the usefulness of gated CT acquisition for the quantification of PET/CT by comparing the different acquisition protocols using a moving hot sphere phantom. 
Respiratory Gated PET/CT

Phantom and Motion Tables

The National Electrical Manufacturers Association (NEMA) 2001 International Electrotechnical Commission (IEC) phantom (Data Spectrum Corp., Hillsborough, NC) consisting of a quasicylindrical cavity $(280 \times 210 \times 180 \mathrm{~mm})$ and six spheres (Model ECT/IEC -BODY/P) was used for this study. The spheres were 10, 13, 17, 22, 28, and $37 \mathrm{~mm}$ in diameter, and their wall thickness was $1 \mathrm{~mm}$. All of the spheres were filled with ${ }^{18} \mathrm{~F}$ solution of $36.7 \mathrm{kBq} / \mathrm{mL}$, and the background was filled with air. The radioactivity was equal to $\mathrm{SUV}=15$. A newly designed motion system was used in this study to simulate respiratory motion. The NEMA phantom was placed on a moving table, and a motor-driven table oscillated with a displacement of $2 \mathrm{~cm}$ in the craniocaudal direction and a frequency of $15 / \mathrm{min}$. The parameters were selected to simulate displacements and respiratory cycles typically observed in normal respiratory motion.

The motion tracking was recorded by a real-time position management (RPM) respiratory gating system (Varian Medical Systems, Palo Alto, CA). The motion was sinusoidal. A trigger was set at a defined phase within the respiratory cycle and initiated the acquisition cycle in gated PET and dynamic CT. Finally, the respiratory cycles were 
Respiratory Gated PET/CT

divided into eight phases.

Data Acquisition

PET/CT scans were acquired in both a nonmoving status (nonmotion) and a moving status (motion) on a Discovery ST Elite (GE Healthcare, Milwaukee, WI). The 16-slice CT scanning parameters were $120 \mathrm{kv}, 30-154 \mathrm{~mA}$, matrix $512 \times 512$, slice thickness $5 \mathrm{~mm}$, and transaxial field of view $500 \mathrm{~mm}$. Gantry rotation was 0.5 sec/rotation for conventional helical CT (HCT), $5.0 \mathrm{sec} /$ rotation for SCT, and 0.5 sec/rotation in the cine mode for ACT and 4DCT. The HCT was performed in a nonmoving status with spheres placed at the center position of movement, which is the midpoint between phases 2 and 3. A $5 \mathrm{~s}$ cine duration time and a $0.45 \mathrm{~s}$ interval time between image reconstructions were used as the cine mode parameters. The total scan length was set to cover the full displacement of the phantom. The ACT data sets were generated based on fixed cine duration averaging. The ACT data sets were generated based on fixed cine duration averaging and used for attenuation correction. CTAC using ACT was mathematically processed with averaged cine CT data, but the ACT images could not be generated and could not be shown by our PET/CT system.

Emission data were acquired in the $3 \mathrm{D}$ mode with $128 \times 128$ matrices 
Respiratory Gated PET/CT

$(5.47 \times 5.47 \times 3.27 \mathrm{~mm})$. The acquisition times per bed position were 3 minutes in static mode and 8 minutes in gated mode. PET was reconstructed using a 3D ordered subsets-expectation maximization (3D-OSEM) algorithm (VUE Point Plus, 2 iterations, 28 subsets, and a post-filter of $6 \mathrm{~mm}$ full width at half maximum (FWHM)).

The PET/CT images in nonmotion scans were reconstructed with a combination of static PET and helical CT data and were used as a standard. The static PET (sPET) in motion was reconstructed with HCT, SCT, and ACT, and the gated PET (gPET) was reconstructed with HCT and 4DCT.

Data Analysis

For the PET data, the maximum radioactivity and the dimensions of each sphere were measured on each central coronal slice using a region of interest (ROI). On the monitor displaying PET images, upper and lower SUV were set to 6.0 and 0.0 , respectively, according to routine clinical use. To measure the maximum counts of the sphere, the circular ROI equal to the known sphere size was placed to delineate the sphere under careful visual observation. The ROI must include the area of highest activity. The dimensions of each sphere were measured by manual delineation of the sphere images during the visual assessment. To evaluate the quantification of the PET 
Respiratory Gated PET/CT

data, RC, \%Counts, and \%Dimensions were calculated by:

$$
\begin{aligned}
& \mathrm{RC}=\mathrm{Ci} / \mathrm{C}_{37} \times 100 \\
& \% \text { Counts }=(\mathrm{Ci}-\mathrm{Cr}) / \mathrm{Cr} \times 100 \\
& \% \text { Dimensions }=(\mathrm{Di}-\mathrm{Dr}) / \mathrm{Dr} \times 100
\end{aligned}
$$

where $\mathrm{Ci}$ and $\mathrm{Di}$ are interest values, $\mathrm{C}_{37}$ is the maximum radioactivity of the $37 \mathrm{~mm}$ sphere in nonmotion, $\mathrm{C}_{\mathrm{r}}$ is that of corresponding size sphere in nonmotion, and the $\mathrm{D}_{\mathrm{r}}$ is actual inside dimensions of each sphere were used.

For the CT images, the dimensions and the CT number of spheres were measured on a center slice of each coronal image using a manually placed ROI that delineated the outside surface of each sphere. The \%Dimensions and \%Numbers of the CT images were calculated to evaluate the CT data as CTAC. The \%Dimensions were defined in the same way as PET data. The CT number of pixels in each sphere was segmentally classified into two categories--water density (from -30 HU to $30 \mathrm{HU}$ ) and noise density (from $-800 \mathrm{HU}$ to $-30 \mathrm{HU}$ )--using the profile curves of the CT images. The \%Numbers was the ratio of the number of pixels with each CT number to that of all pixels in the ROI. As a reference for \%Dimensions and \%Numbers, the actual outside 
Respiratory Gated PET/CT

dimensions and the number of all pixels in the ROI of each sphere were used.

Statistical Analysis

Multiple comparisons of the \%Counts, \%Dimensions, and \%Numbers among the difference acquisition protocols were performed using the Tukey-Kramer method. The levels of significance were set at $\mathrm{p}<0.05$ and $\mathrm{p}<0.01$.

\section{RESULTS}

Comparison of PET Counts

The RC curve of each examination is shown in figure 1. The top curve represents the RC of the nonmotion static PET. The gradual decrease of the RC was observed in spheres with diameters of $22 \mathrm{~mm}$ or smaller. For the RCs in motion, the sPET-HCT decreased markedly, and the $10 \mathrm{~mm}$ sphere was not observed. The RCs of sPET-SCT were lower than those of sPET-HCT. The RCs of sPET-ACT were similar to those of sPET-SCT. On the other hand, the gPET-HCT showed improvement of RCs, and the gPET-4DCT improved further. Figure 2 shows both CT and PET images of 
nonmotion sPET-HCT, motion sPET-SCT and motion gPET-4DCT.

The PET count of each sphere in the different acquisition protocols was compared with that of sPET-HCT in the nonmotion status and was expressed as \%Counts (table 1). The \%Counts of the sPET-HCT, the sPET-SCT, and the sPET-ACT decreased, especially in the small spheres; their respective mean \%Counts were $-43.8 \%,-51.4 \%$, and $-49.5 \%$, respectively. On the other hand, the degrees of decrease in the \%Counts of the gPET-HCT and the gPET-4DCT were mild relative to that of the sPET. The mean \%Counts of the gPET-HCT and the gPET-4DCT improved to $-30.1 \%$ and $-15.2 \%$, respectively.

The \%Counts of the gPET-HCT and the gPET-4DCT in each respiratory phase are shown in figure 2. In gPET-HCT, the \%Counts of the central phases (phases 2, 3, 6, and 7) were smaller than those of edge phases (phases 1, 4, 5, and 8). The sphere position during the HCT scan was the midpoint between phases 2 and 3. On the other hand, the \%Counts of edge phases (phases 1, 4, 5, and 8) were smaller than those of central phases (phases 2, 3, 6, and 7) in gPET-4DCT. Figure 3 shows the gated PET and CT images in each respiratory phase. In the gPET-HCT images, the distribution of radioactivity was not homogeneous. The hot area was located in the center position of the respiratory movement where the sphere was placed during HCT scanning. In 
Respiratory Gated PET/CT

gPET-4DCT, the distribution of radioactivity was homogeneous.

Comparison of the Sphere Dimensions

The sphere dimensions of the CT and PET images are shown in table 2 and figure 2. The \%Dimensions of sPET-HCT (89.7\%), sPET-SCT (82.4\%), and sPET-ACT (84.5\%) were significantly higher than those of nonmotion $(\mathrm{p}<0.01)$, gPET-HCT $(\mathrm{p}<$ 0.05), and gPET-4DCT $(\mathrm{p}<0.05)$. However, those of gPET-HCT $(28.1 \%)$ and gPET-4DCT $(24.9 \%)$ were close to the standard values. Considering the sphere dimensions on the CT images, the dimensions of the SCT were significantly higher than the standard values $(\mathrm{p}<0.01)$. Those of the 4DCT were also close to the standard values.

Comparison of Sphere Density of CT images

The \%Numbers of the water density and that of the noise density are shown in figure 5 . The \%Numbers of the water density of SCT in motion was significantly lower than that of the nonmotion HCT ( $p<0.01)$. Those of 4DCT also decreased significantly $(\mathrm{p}<0.01)$, while the degree of decrease was milder than in those of SCT. The $\%$ Numbers of the noise density of SCT in motion significantly increased $(\mathrm{p}<0.01)$. 
Respiratory Gated PET/CT

Although that of 4DCT also increased significantly, it was lower than that of SCT ( $<<$ 0.01). The smaller the sphere size, the greater the increase in the $\%$ Numbers of the noise density.

The $\%$ Numbers of 4DCT in each respiratory phase is shown in figure 6 . In 4DCT, the mean \%Numbers of the water density decreased and that of the noise density increased in the central phases. However, those of both the water and noise densities in the edge phases were close to those of the HCT in nonmotion.

\section{DISCUSSION}

This study examined the usefulness of gated CT acquisition for the quantification of gated PET/CT by comparing a different acquisition protocol using a moving hot sphere phantom.

In nonmotion, the RCs of the sPET-HCT were more than $90 \%$ in the spheres with diameters of at least $17 \mathrm{~mm}$. In motion, the PET counts decreased with motion. Furthermore, the smaller the sphere size, the more the PET counts decreased. Pevsner et al (11) also compared the maximum radioactivity of spheres in motion with a 
displacement of $2 \mathrm{~cm}$ with those in nonmotion. They reported that the RCs of $13 \mathrm{~mm}$ and $22 \mathrm{~mm}$ spheres in motion were underestimated by as much as $75 \%$ and $40 \%$, respectively. Okubo et al (12) examined the quantification of PET/CT using a moving phantom oscillating with a displacement of $10 \mathrm{~mm}, 20 \mathrm{~mm}$, and $30 \mathrm{~mm}$. They reported that the SUV max of $22 \mathrm{~mm}$ and $28 \mathrm{~mm}$ spheres also decreased when the displacement was $30 \mathrm{~mm}$. The PET counts in motion decreased because the PET counts of the sphere were distributed into the widely smeared area determined by both the sphere diameter and displacement distance. This phenomenon was caused by the overlap in PET emission data interacting between the sphere diameter and the displacement distance.

The \%Counts of the sPET-HCT, sPET-SCT, and sPET-ACT markedly decreased in motion, while those of the gPET-HCT and gPET-4DCT mildly decreased. The gated PET acquisition is thus considered to improve the dispersion of radioactivity in comparison with the static PET acquisition. Nehmeh et al (8) evaluated the effect of gated acquisition on motion-induced underestimation using an oscillating point source. They reported that the gated acquisition improved the distribution of radioactivity. Vines et al (13) compared the static PET acquisition in motion to the gated PET acquisition in motion. They reported that the PET counts of gated PET in motion, compared with those of static PET in motion, were closer to those of static PET in nonmotion. These 
results suggest that the gated acquisition of PET is necessary to obtain an accurate PET count in motion. Although the gPET-4DCT improved quantitative accuracy, it could not obtain complete recovery in comparison to the sPET-HCT in nonmotion in this study. The difference may be due to the residual movement of the spheres during respiratory phases in spite of the gated acquisition. Another possibility is the short acquisition time of $\operatorname{gPET}(1 \mathrm{~min} /$ phase $\times 8$ phases $=8 \mathrm{~min})$, resulting in an increase of statistical variance. Another possibility is the short acquisition time of gPET ( $1 \mathrm{~min} / \mathrm{phase})$ resulting in an increase of statistical variance. This variance is also considered to result in fluctuation of the RC curve and \%Counts of the 37- and 28-mm sphere in gPET-HCT, as shown in Table 1 and Figure 1.

The \%Dimensions of motion sPET were significantly higher than those of nonmotion sPET. The gated acquisition of PET minimizes the overestimation of the sphere dimensions in motion, while the \%Dimensions of gPET were still higher than those of nonmotion sPET. Although a respiratory cycle was divided into 8 phases in this study, residual movement of spheres in a phase is considered to result in image blurring. In gPET, an increase of statistical variance of radioactivity due to a short acquisition time may be another cause of increased \%Dimensions. In Table 2, the \%Dimensions of the $10-\mathrm{mm}$ sphere in both gPET-HCT and gPET-4DCT were 
strangely smaller than those of larger spheres. We believe the reason for this is that the contour of a sphere with such a small diameter cannot be clearly visualized due to the underestimation of radioactivity. The limited spatial resolution of our PET system underestimates radioactivity of small spheres, and the $\mathrm{RC}$ of the $10^{-} \mathrm{mm}$ sphere was about $60 \%$. Furthermore, residual movement of spheres in a phase probably caused further underestimation of radioactivity due to a smearing effect, in spite of the gated acquisition. These factors more strongly affected the sPET images in motion. The 10-mm spheres in motion sPET images could not be visualized at all.

CTAC is also important for the quantification of PET. Some clinical studies have reported that the misregistration between PET emission data and CTAC caused the underestimation of PET counts. Erdi et al (4) reported that misregistration between PET emission data and CTAC resulted in SUV variability of up to $30 \%$. Some studies have reported that the application of both SCT and ACT for CTAC was useful for improving quantification $(6,7)$. However, the present results showed that the underestimation of PET counts could not be improved even when SCT or ACT was used for CTAC. Both SCT and ACT include the motion of several respiratory cycles and provide density-averaged CT images because they were performed with a long rotation time and fixed cine duration images, respectively. Because SCT decreased the $\%$ Numbers for 
water density, while conversely increasing that for noise density, the CTAC of SCT might therefore not reflect the actual attenuation map of the sphere. Chi et al (7) proposed an ACT using 4DCT for attenuation correction with static PET data. They reported that it reduced $\mathrm{PET} / \mathrm{CT}$ misregistration by matching the temporal resolution between PET and CT data. Although the misregistration between PET emission data and CTAC of SCT and ACT was minimized, the PET counts did not improve when either SCT or ACT was used for CTAC. In addition, neither sPET-SCT nor sPET-ACT improved the underestimation of radioactivity or the overestimation of tumor volume in the static PET in motion. As a result, both SCT and ACT were considered inadequate for CTAC.

The gated PET with 4DCT improved the quantification of PET images in respiratory motion. Although the 4DCT images showed slightly lower water density than HCT, the \%Counts for gPET-4DCT were better than those for gPET-HCT. This is thought to result from the better image registration of 4DCT to gated PET images at each respiratory phase. In a recent study with gated PET acquisition, Nagel et al (14) evaluated the potential of 4DCT to improve PET counts in comparison with HCT. They found that 4DCT resulted in improved PET counts of up to $28 \%$. In the present study, the radioactivity distribution of the gPET-HCT images was not homogeneous. The 
Respiratory Gated PET/CT

highest radioactive area in the sphere was observed in a limited part of the sphere where the PET images and the CTAC were spatially matched. The highest radioactive area in the sphere was located in a different part from phase to phase. In gPET-HCT, the sphere position of PET was different among phases, while that of HCT was fixed at the center position during movement. The \%Counts of the central phases where PET images were well registered to CT images were superior to those of the edge phases. On the other hand, the \%Counts of the central phases were inferior to those of the edge phase in the gPET-4DCT. Although the PET images and CTAC showed good registration at each phase in gPET-4DCT, the residual movement of spheres in a phase of the edge phases was smaller than that of the central phases. The differences in the degree of blurring are considered to cause the different \%Counts among phases. However, the distribution of radioactivity in gPET-4DCT was relatively homogeneous, and the difference in the \%Counts among phases was minimal. Therefore, the gated acquisition of both PET and CT is considered the best acquisition protocol in motion in this study.

The phantom background in this study was filled with air because we were simulating human lung tumor. In the case of abdominal tumors with respiratory motion, we must consider the result when the phantom background is filled with water by 
bibliographic cinsideration. The density inside the phantom must be homogeneous and around water density because the phantom consists of only water and the phantom wall. We can ignore the partial volume effect on the CT images of the sphere. Thus the $\%$ Numbers should be $100 \%$ water density regardless of the different CT protocol. Furthermore, misregistration between PET images and CT images are also supposed to be ignored. These factors are thought to increase the PET counts of spheres due to the improved accuracy of attenuation correction. The differences in \%Counts among the different CT protocols are thought to disappear too. Vines et al. (13) estimated the difference in PET counts among static and gated acquisition in motion and static acquisition in nonmotion with corrected helical CT attenuation. The difference in PET counts between sPET-HCT in nonmotion and gPET-HCT in motion were equal to or less than $10 \%$ for spheres more than $17 \mathrm{~mm}$ in diameter. This is in agreement with the result for gPET-4DCT in the present study.

The gated PET data in this study were divided into eight phases, and the acquisition time of each phase was thus one minute. The acquisition time of each phase determined by the number of phases and the total acquisition time would have an effect on the quantification of PET data. Motion-induced underestimation would also be different for each tumor size at different displacement distances and sphere background 
Respiratory Gated PET/CT

ratios of radioactivity. The respiratory motions of patients are not usually regular, though those in the current phantom study were regular. Further examinations under several different conditions and with clinical trials are required to confirm the general usefulness of gPET-4DCT for quantification. The radiation dose is another problem. The radiation dose of $4 \mathrm{DCT}$ with about 5 seconds of cine duration has been reported to be between 23 and $70 \mathrm{mGy}(7,15)$. The dose is approximately six times the dose of a single helical CT and is considered to be too high to be used in a routine diagnostic procedure (16). We must investigate methods to minimize the radiation dose of 4DCT.

\section{CONCLUSIONS}

In respiratory motion, PET acquisition in the static mode underestimated the radioactivity and overestimated the dimensions. Neither SCT nor ACT improved these errors. PET acquisition in the gated mode improved the quantification of PET/CT images, though gated PET acquisition using 4DCT is necessary for further improvement. 
Respiratory Gated PET/CT

\section{ACKNOWLEDGMENTS}

The authors thank Mr. Hirofumi Kawakami (GE Healthcare Japan) and Mr. Naoyuki Tamamura (Nihon MediPhysics) for their technological assistance. 


\section{REFERENCE}

1. Weber WA and Figlin R. Monitoring Cancer Treatment with PET/CT: Does It Make a Difference? J Nucl Med. 2007;48:36-44.

2. Goerres GW, Kamel E, Heidelberg TN, Schwitter MR, Burger C, von Schulthess GK. PET-CT image co-registration in the thorax: influence of respiration. Eur $\mathrm{J}$ Nucl Med. 2002;29:351-60.

3. Cohade C, Osman M, Marshall LT, Wahl RN. PET-CT: accuracy of PET and CT spatial registration of lung lesions. Eur J Nucl Med Mol Imaging. 2003;30:721-6.

4. Erdi YE, Nehmeh SA, Pan T, Pevsner A, Rosenzweig KE, Mageras G et al. The CT motion quantitation of lung lesions and its impact on PET-measured SUVs. J Nucl Med. 2004;45:1287-92.

5. Lagerwaard FJ, Van Sornsen de Koste JR, Nijssen-Visser MR, Schuchhard-Schipper RH, Oei SS, Munne A, et al. Multiple "slow" CT scans for incorporating lung tumor mobility in radiotherapy planning. Int. J. Radiat. Oncol., Biol. Phys. 2001;51:932-7.

6. Nye JA, Esteves F, Votaw JR. Minimizing artifacts resulting from respiratory and cardiac motion by optimization of the transmission scan in cardiac PET/CT. Med 
Phys. 2007; 34: 1901-06.

7. Chi PC, Mawlawi O, Nehmeh SA, Erdi YE, Balter PA, Luo D et al. Design of respiratory averaged $\mathrm{CT}$ for attenuation correction if the PET data from PET/CT. Med Phys 2007; 34: 2039-2047.

8. Nehmeh SA, Erdi YE, Ling CC, Rosenzweig KE, Squire OD, Braban LE, et al. Effect of respiratory gating on reducing lung motion artifacts in PET imaging of lung cancer. Med Phys. 2002;29:366-71.

9. Nehmeh SA, Erdi YE, Pan T, Yorke E, Mageras GS, Rosenzweig KE, et al. Quantitation of respiratory motion during 4D-PET/CT acquisition. Med Phys. 2004;31:1333-8.

10. Pan T, Lee TY, Rietzel E, Chen GT. 4D-CT imaging of a volume influenced by respiratory motion on multi-slice CT. Med Phys. 2004;31:333-40.

11. Pevsner A, Nehmeh S A, Humm J L, et al: Effect of motion on tracer activity dertemination in CT attenuation corrected PET images: A lung phantom study. Med Phys 2005; 32: 2358-62.

12. Okubo M, Nishimura Y, Nakamatsu K, et al: Static and moving phantom studies for radiation treatment planning in a positron emission tomography and computed tomography (PET/CT) system. Ann Nucl Med 2008; 22: 579-86. 
Respiratory Gated PET/CT

13. Vines D C, Keller H, Hoisak J D P, et al: Quantitative PET comparing gated with nongated acquisitions using a NEMA phantom with respiratory-simulated motion. J Nucl Med Technol 2007; 35: 246-51.

14. Nagel C C A, Bosmans G, Dekker A L A J, et al: Phased attenuation correction in respiratory correlated computed tomography/positron emitted tomography. Med. Phys 2006; 33: 1840-7.

15. Pan T, Mawlawi O, Nehmeh SA, Erdi YE, Luo D, Liu HH, et al. Attenuation correction of PET images with respiration-averaged CT images in PET/CT. J Nucl Med. 2005; 46: 1481-7.

16. Slotman BJ, Lagerwaard FJ, Senan S. 4D imaging for target definition in stereotactic radiotherapy for lung cancer. Acta Oncol. 2006; 45: 966-72. 


\section{LEGENDS FOR FIGURES}

Figure 1 The RC curves of differences in PET/CT acquisitions. The maximum radioactivity of the $37 \mathrm{~mm}$ sphere in nonmotion $\mathrm{SPET} / \mathrm{HCT}$ is used as a reference. The smaller the sphere size, the lower the RC. In motion SPET, a sphere with a diameter of $10 \mathrm{~mm}$ cannot be visualized.

Figure $2 \mathrm{CT}$ and PET images of $37 \mathrm{~mm}$ sphere. In comparison with the nonmotion HCT image (A), the SCT image blurs due to motion (B). The 4DCT image shows only a little blurring (C). In comparison with the PET image of sPET-HCT in nonmotion (D), that of the sPET-SCT appears to be blurred (E). The gPET-4DCT appears to be less blurred (F).

Figure 3 The \%Counts of the gated PET in each respiratory phase. A. gPET-HCT, B. gPET-4DCT.

Figure 4 Gated PET and CT images in respiratory motion. Coronal images of the 37 mm sphere in each respiratory phase are shown; (A) HCT, (B) gPET-HCT, (C) 4DCT and (D) gPET-4DCT. Broken lines indicate the center position of the displacement. The radioactivity distribution in the sphere of the gPET-HCT was not homogeneous. The hot area in the sphere differs among 
Respiratory Gated PET/CT

phases. That of gPET-4DCT was homogeneous.

Figure 5 The $\%$ Numbers of pixels with CT numbers for water density and noise density in different CT scan modes.

Figure 6 The \%Numbers of pixels with CT numbers for water density and noise density in each respiratory phase of $4 \mathrm{DCT}$. The $\%$ Numbers shows the mean of all spheres. 
Respiratory Gated PET/CT

Table 1 Comparison of PET counts in different PET/CT protocols. The numbers are the \%Counts in comparison to the PET counts of sPET-HCT in the nonmotion status.

\begin{tabular}{|c|c|c|c|c|c|c|c|}
\hline & \multicolumn{6}{|c|}{ Sphere diameters (mm) } & \multirow[b]{2}{*}{ mean } \\
\hline & 10 & 13 & 17 & 22 & 28 & 37 & \\
\hline sPET-HCT & - & -65.3 & -44.4 & -29.8 & -15.1 & -8.0 & $-43.8^{*}$ \\
\hline sPET-SCT & - & -77.1 & -51.7 & -38.7 & -25.2 & -15.7 & $-51.4^{* *}$ \\
\hline sPET-ACT & - & -63.50 & -52.37 & -41.46 & -24.58 & -15.21 & $-49.5^{* * *}$ \\
\hline gPET-HCT & -70.5 & -26.9 & -22.1 & -18.4 & -22.5 & -20.2 & -30.1 \\
\hline gPET-4DCT & -39.0 & -17.7 & -11.6 & -9.5 & -8.0 & -5.8 & -15.2 \\
\hline
\end{tabular}

* The mean \%Counts of the sPET-HCT were significantly lower than those of gPET-4DCT $(\mathrm{p}<0.01)$.

** The mean \%Counts of the sPET-SCT were significantly lower than those of gPET-HCT $(\mathrm{p}<0.05)$ and gPET-4DCT $(\mathrm{p}<0.01)$. *** The mean \%Counts of the sPET-ACT were significantly lower than those of gPET-HCT $(\mathrm{p}<0.05)$ and gPET-4DCT $(\mathrm{p}<0.01)$. 
Respiratory Gated PET/CT

Table 2 Comparison of sphere dimensions on PET and CT images. The numbers are the $\%$ Dimensions in comparison to the actual dimensions.

\begin{tabular}{|c|c|c|c|c|c|c|c|c|c|}
\hline & & & \multicolumn{6}{|c|}{ Sphere diameters (mm) } & \multirow[b]{2}{*}{ Mean } \\
\hline & & & 10 & 13 & 17 & 22 & 28 & 37 & \\
\hline \multirow{7}{*}{ PET } & Nonmotion & sPET-HCT & 11.2 & 16.5 & 14.7 & 4.8 & 2.4 & 0.6 & 8.4 \\
\hline & Motion & sPET-HCT & - & 173 & 151 & 109 & 50.2 & 54.5 & $89.7^{*}$ \\
\hline & & sPET-SCT & - & 172 & 125 & 90 & 49.1 & 57.8 & $82.4^{* *}$ \\
\hline & & & & & & & & & \\
\hline & & sPET-ACT & - & 173 & 130 & 97 & 51.2 & 55.6 & $84.5^{* * *}$ \\
\hline & & gPET-HCT & 17.1 & 44.3 & 48.2 & 27.3 & 19.7 & 11.7 & 28.1 \\
\hline & & gPET-4DCT & 8.2 & 46 & 38.5 & 22.2 & 23.4 & 11.1 & 24.9 \\
\hline \multirow{3}{*}{$\mathrm{CT}$} & Nonmotion & HCT & 14.6 & 11.4 & 13.7 & 11.5 & 5.8 & 0.2 & 9.5 \\
\hline & Motion & $\mathrm{SCT}$ & 208.1 & 174.5 & 149 & 117.4 & 80.6 & 63.1 & $132.1^{* * * *}$ \\
\hline & & $4 \mathrm{DCT}$ & 62.4 & 61.5 & 58.2 & 44.3 & 30.5 & 28.9 & 30.1 \\
\hline
\end{tabular}

* The mean \%Dimensions of the sPET-HCT were significantly higher than those of nonmotion sPET-HCT $(\mathrm{p}<0.01)$, gPET-HCT $(\mathrm{p}<0.05)$, and gPET-4DCT $(\mathrm{p}<0.01)$. ** The mean \%Dimensions of the sPET-SCT were significantly higher than those of nonmotion sPET-HCT $(\mathrm{p}<0.01)$, gPET-HCT $(\mathrm{p}<0.05)$, and gPET-4DCT $(\mathrm{p}<0.05)$. *** The mean $\%$ Dimensions of the sPET-ACT were significantly higher than those of 
Respiratory Gated PET/CT

nonmotion sPET-HCT $(\mathrm{p}<0.01)$, gPET-HCT $(\mathrm{p}<0.05)$, and gPET-4DCT $(\mathrm{p}<0.05)$.

**** The mean $\%$ Dimensions of the SCT were significantly higher than those of

nonmotion HCT $(\mathrm{p}<0.01)$ and 4DCT $(\mathrm{p}<0.01)$. 
Figure 1

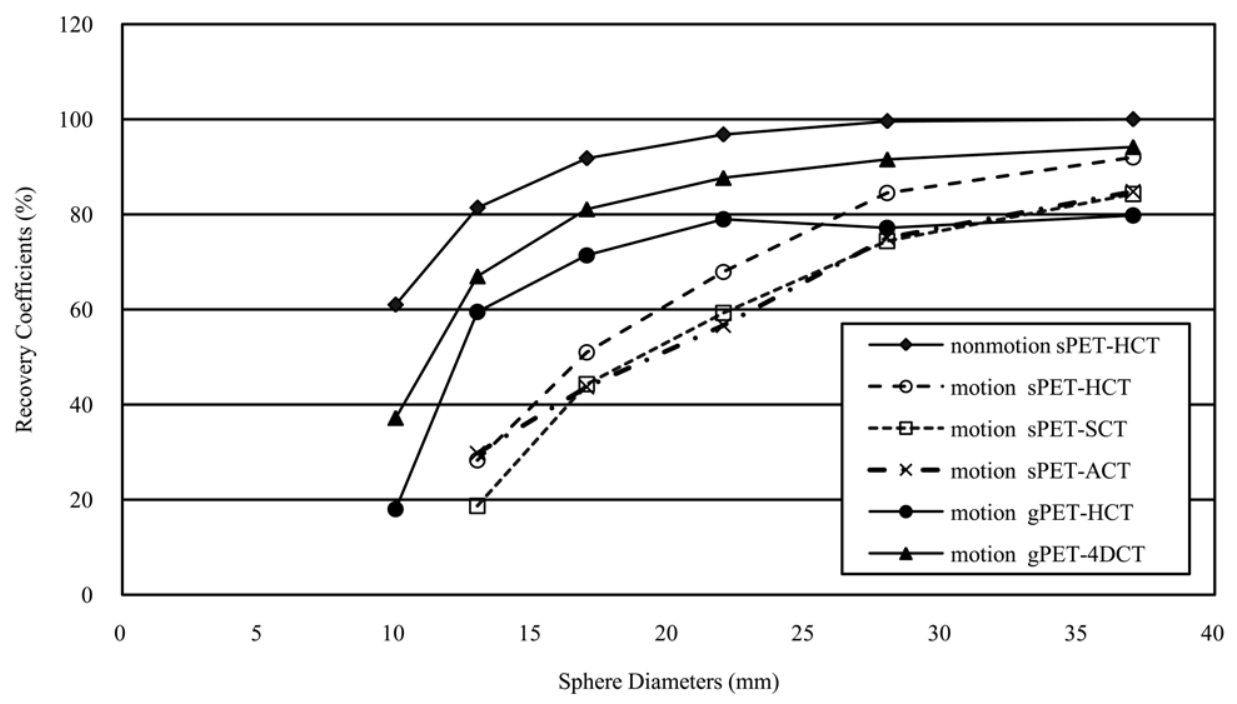


Figure 2

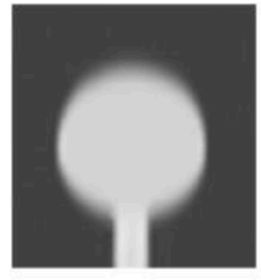

(a)

(d)

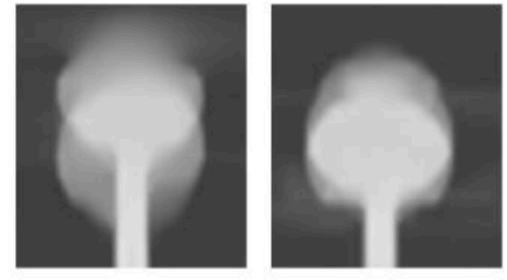

(b)

(c)

(e)

(f) 
Figure 3

(A)

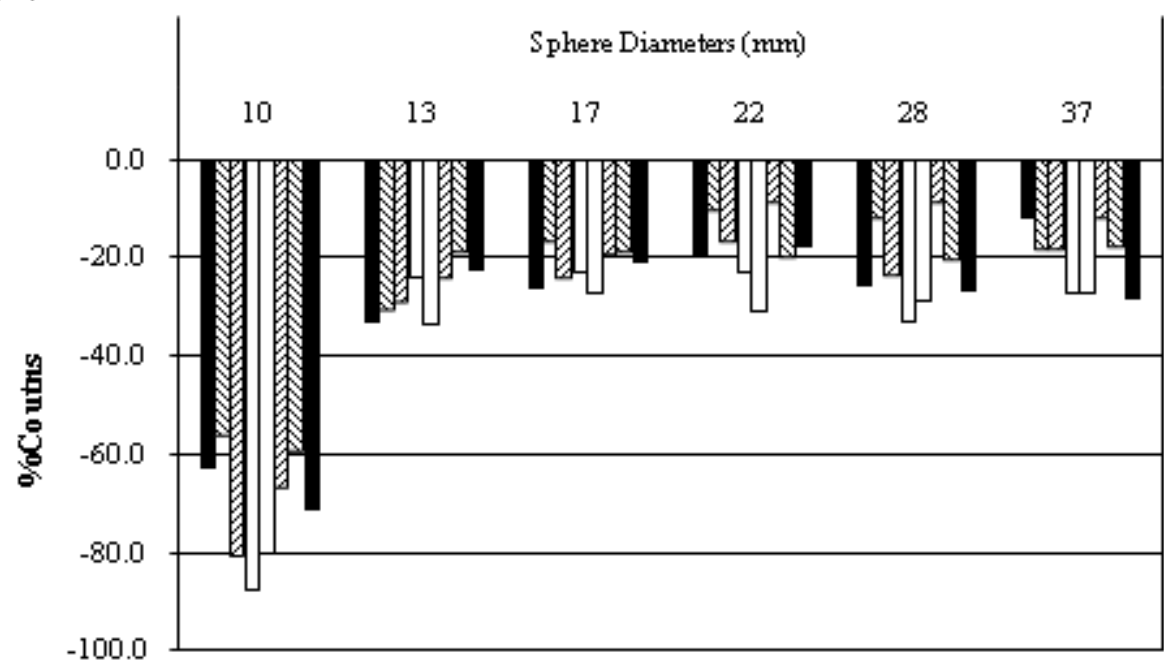

- phase 1

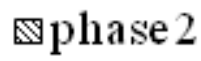
ஐphase 3 $\square$ phase 4 $\square$ phase 5 刃phase 6 \&phase 7 - phase 8

(B)

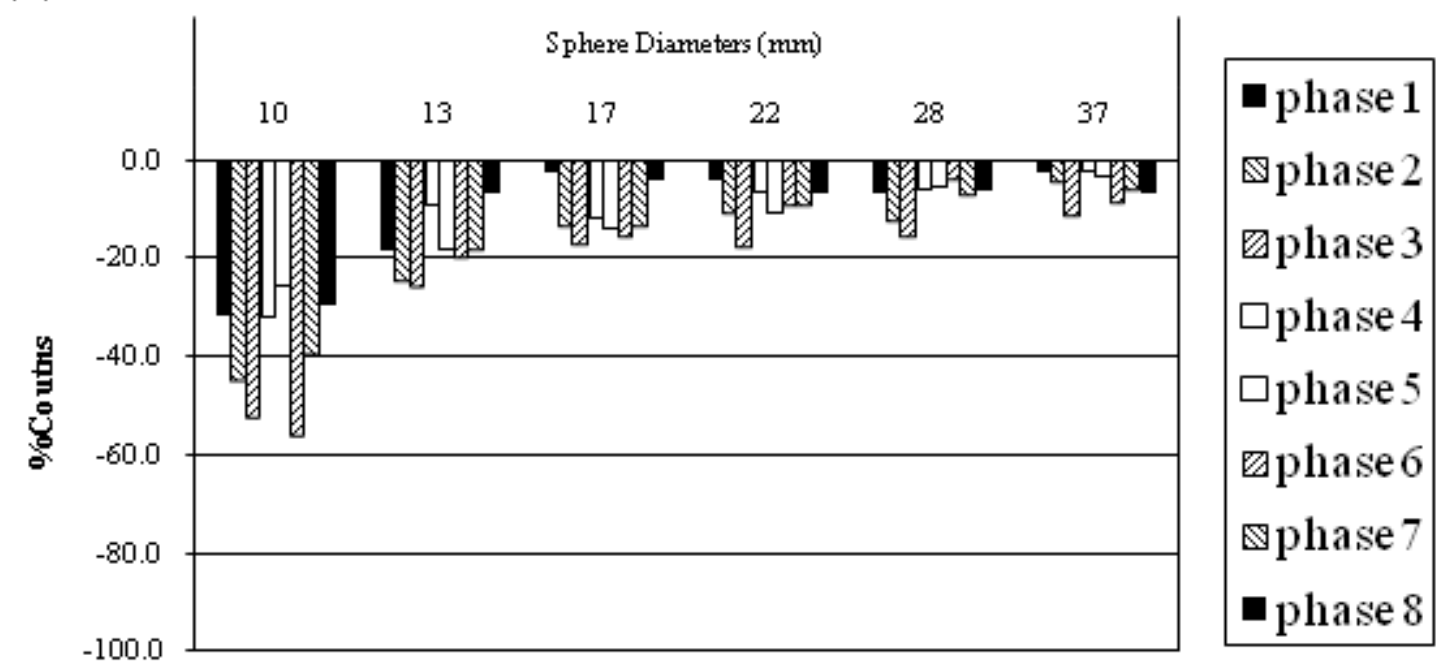


Figure 4

(a)

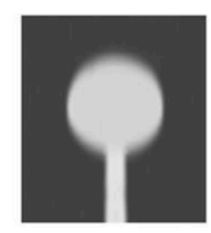

(b)

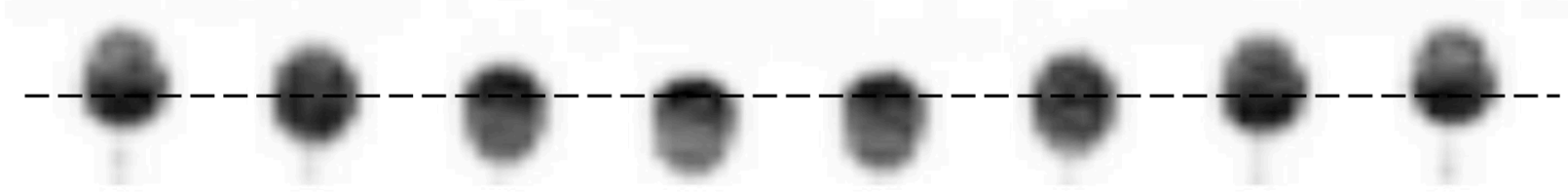

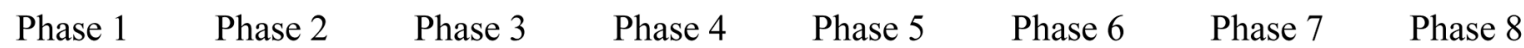

(c)

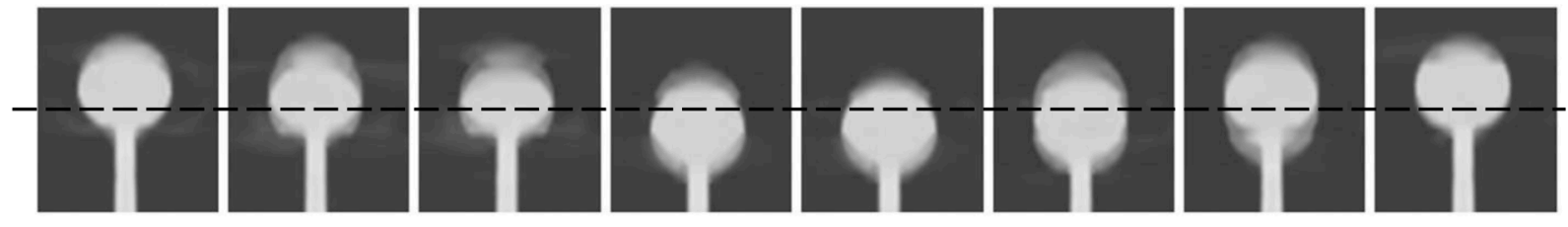

(d)

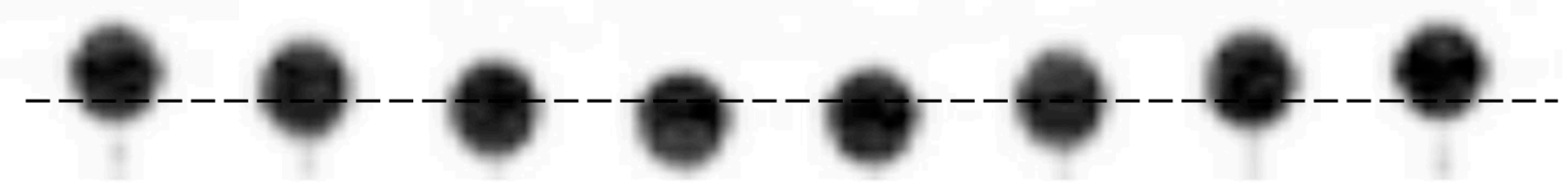

$\begin{array}{llllllll}\text { Phase } 1 & \text { Phase } 2 & \text { Phase } 3 & \text { Phase } 4 & \text { Phase } 5 & \text { Phase } 6 & \text { Phase } 7 & \text { Phase } 8\end{array}$ 
Figure 5

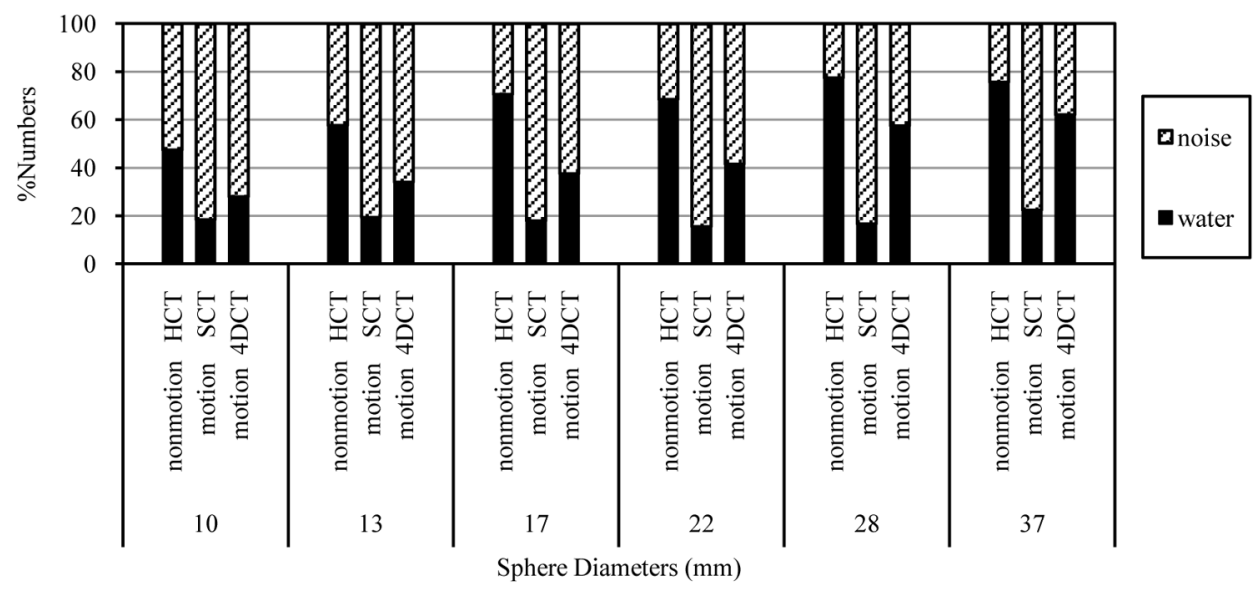


Figure 6

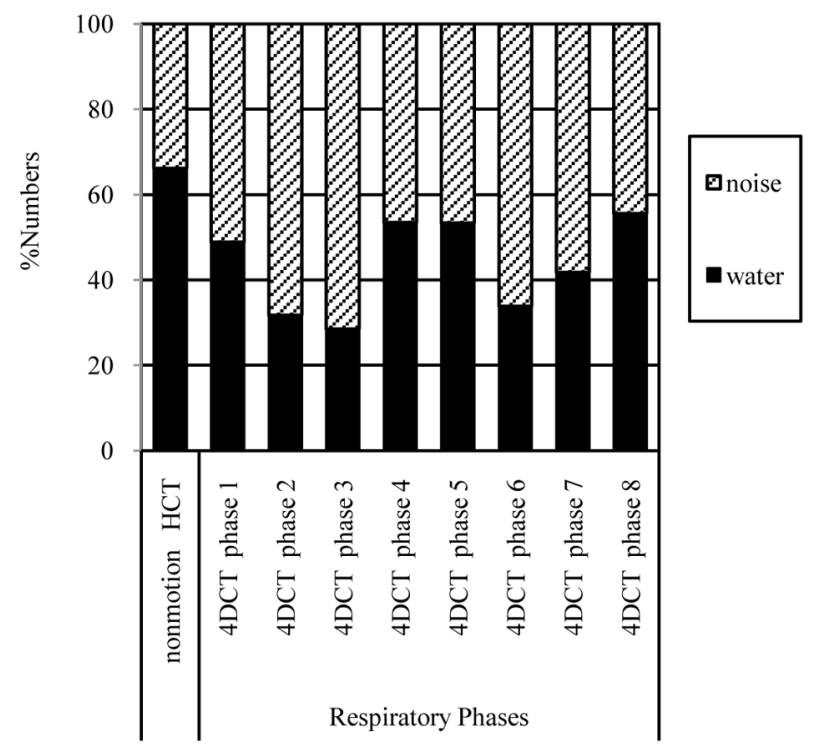

